\title{
Spin-polarized Shapiro steps and spin-precession-assisted multiple Andreev reflection
}

\author{
C. Holmqvist, ${ }^{1}$ M. Fogelström, ${ }^{2}$ and W. Belzig ${ }^{1}$ \\ ${ }^{1}$ Fachbereich Physik, Universität Konstanz, D-78457 Konstanz, Germany \\ ${ }^{2}$ Department of Microtechnology and Nanoscience-MC2, Chalmers University of Technology, SE-412 96 Göteborg, Sweden
}

(Received 25 February 2013; revised manuscript received 30 June 2014; published 28 July 2014)

\begin{abstract}
We investigate the charge and spin transport of a voltage-biased superconducting point contact coupled to a nanomagnet. The magnetization of the nanomagnet is assumed to precess with the Larmor frequency $\omega_{L}$ when exposed to ferromagnetic resonance conditions. The Larmor precession locally breaks the spin-rotation symmetry of the quasiparticle scattering and generates spin-polarized Shapiro steps for commensurate Josephson and Larmor frequencies that lead to magnetization reversal. This interplay between the ac Josephson current and the magnetization dynamics occurs at voltages $|V|=\hbar \omega_{L} / 2 e n$ for $n=1,2, \ldots$, and the subharmonic steps with $n>1$ are a consequence of multiple Andreev reflection (MAR). Moreover, the spin-precession-assisted MAR generates quasiparticle scattering amplitudes that, due to interference, lead to current-voltage characteristics of the dc charge and spin currents with subharmonic gap structures displaying an even-odd effect.
\end{abstract}

DOI: 10.1103/PhysRevB.90.014516 PACS number(s): 75.76.+j, 74.50.+r, 75.50.Xx, 75.78. $-\mathrm{n}$

\section{INTRODUCTION}

The combination of mesoscopic superconductivity and spintronics has led to the development of superconducting spintronics [1]. Such spintronics devices rely on the existence of superconducting spin-triplet correlations, whose spin properties determine the behavior of these devices. So far, experiments on devices comprising spin-triplet superconductors [2-4] have been scarce, although several exotic effects have been predicted [5-7]. Instead, spin-triplet correlations have been created from spin-singlet correlations originating from conventional superconductors with the help of ferromagnets [8-11]. Up to now, these devices have consisted mainly of superconductor-ferromagnet-superconductor junctions with noncollinear magnetizations whose properties cannot easily be modified after the fabrication stage. However, tunable devices are desirable for future spintronics applications, and the search for such is currently an ongoing quest.

In this paper, we present a theoretical investigation showing how resonant spin supercurrents controlled by a bias voltage can be used to manipulate the magnetization direction and even cause magnetization reversal of a nanomagnet coupled to a superconducting tunnel junction. Instead of a superconducting hybrid junction with a static magnetization [12-18], we consider a junction with a time-dependent one [19-21]. The lowest-energy magnetization excitation can be accessed by the application of an external magnetic field, which starts a precession of the magnetization around the direction of the field [22-25]. This ferromagnetic resonance (FMR) mode can also be achieved by a coupling between the magnetization and the Josephson effect: The ac Josephson current generates an oscillating magnetic field that resonantly excites the magnetization precession [26-28]. The coupling between magnetization dynamics and a spatially dependent superconducting order parameter was experimentally studied in tunnel junctions where the width $W$ was larger than the superconducting coherence length $\xi_{0}$ [27]. This coupling was detected as a rectification of the ac Josephson charge current. However, in narrower tunnel junctions, such as superconducting quantum point contacts (SQPC) [29,30], for which $W<\xi_{0}$, the spin-rotation symmetry of the superconducting order parameter may be broken locally, and one may anticipate that spin-dependent modifications to the superconducting correlations will influence the transport properties [31,32].

Here, we study such a coupling between the ac Josephson effect and the magnetization dynamics of an SQPC containing a nanomagnet, e.g., a ferromagnetic nanoparticle [26] or a single-molecule magnet [33-37]. A bias voltage $V$ is applied across the junction, and the spin of the nanomagnet is set into precession using standard FMR techniques whose effects are captured by the introduction of an effective external magnetic field $\boldsymbol{H}$ that generates a Larmor precession of the nanomagnet's spin with frequency $\omega_{L}$ [Figs. 1(a) and 1(b)]. The Larmor precession creates scattering processes such that quasiparticles traversing the junction may absorb (emit) a precession quantum while undergoing a spin flip from $\downarrow$ ( $\uparrow)$ to $\uparrow(\downarrow)$. The locally broken spin-rotation symmetry generates multiple Andreev reflection (MAR) processes [see examples in panels (c) and (d) of Fig. 1], that lead to a rich subgap structure of the current-voltage characteristics of the dc charge and spin currents. Moreover, at certain voltages, a phase locking between the Larmor precession and the ac Josephson effect occurs that generates Shapiro-like resonances. The phase locking couples the lead condensates via tunneling processes that are exemplified in panel (e) of Fig. 1. Interestingly, instead of giving dc charge contributions as in Ref. [27], we predict that the Shapiro resonances generate dc spin supercurrents that are polarized in the plane perpendicular to $\boldsymbol{H}$. It was shown in Ref. [38] that an oscillating voltage bias can generate spin-polarized Shapiro steps in the presence of a precessing spin. Here, however, we show that a dc voltage bias is sufficient for obtaining spin-polarized Shapiro steps that in turn exert a spin-transfer torque on the spin $S$ that may reverse its direction on subnanosecond time scales [Fig. 1(f)].

The paper is organized as follows. In Sec. II, we present the approach which is based on nonequilibrium Keldysh-Green's function techniques. We then proceed to report results for charge currents in Sec. III and for the spin-polarized Shapiro steps and their corresponding spin-transfer torques in Sec. IV before we conclude in Sec. V. Appendices A, B, and C contain details of the approach, analytic results obtained in 


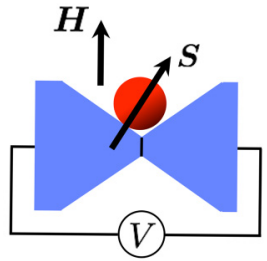

(a)

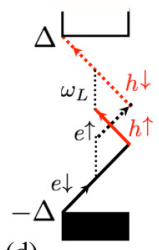

(d)

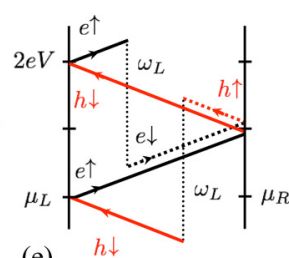

(e)

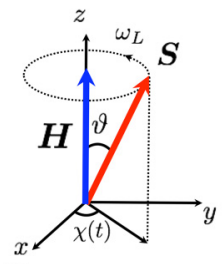

(b)

(c)
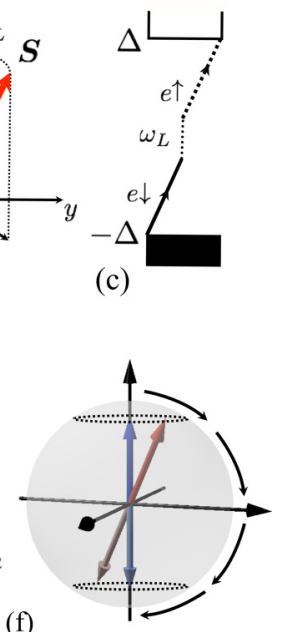

(f)
$\boldsymbol{H}$ that affects the spin according to $\mathcal{H}_{B}=-\gamma \boldsymbol{S} \cdot \boldsymbol{H}$, where $\gamma$ is the gyromagnetic ratio. In the case of a classical spin, this Hamiltonian leads to the equation of motion $d \boldsymbol{S} / d t=$ $-\gamma \boldsymbol{S} \times \boldsymbol{H}$. If $\boldsymbol{H}$, which here is taken to be along the $z$ axis, is applied at angle $\vartheta$ with respect to spin $\boldsymbol{S}$, the solution to the spin's equation of motion describes a precessional motion of $\boldsymbol{S}$ around the direction of $\boldsymbol{H}$ with the Larmor frequency $\omega_{L}=\gamma|H|$. For later convenience, we introduce angle $\chi$ that is formed between the $x$ axis and the projection of $S$ onto the $x y$ plane, see Fig. 1(b). Due to the Larmor precession, this angle is given by $\chi(t)=\chi_{0}+\omega_{L} t$, where $\chi_{0}$ is the initial angle. With these parameters, the spin describes the motion $S=$ $S \boldsymbol{e}_{S}(t)$, where $\boldsymbol{e}_{S}(t)=\left[\cos \chi(t) \sin \vartheta \boldsymbol{e}_{x}+\sin \chi(t) \sin \vartheta \boldsymbol{e}_{y}+\right.$ $\left.\cos \vartheta \boldsymbol{e}_{z}\right]$. The nanomagnet's spin generates a time-dependent exchange field affecting the quasiparticles tunneling in the direction $\pm k$ across the junction that can be incorporated into a phenomenological tunnel Hamiltonian [19,39],

$$
\mathcal{H}_{T}=\sum_{k \sigma ; k^{\prime} \sigma^{\prime}} c_{L, k \sigma}^{\dagger} V_{k \sigma ; k^{\prime} \sigma^{\prime}} c_{R, k^{\prime} \sigma^{\prime}}+\text { H.c. },
$$

with spin $S$. A bias voltage $V$ is applied across the junction that is exposed to an effective magnetic field $\boldsymbol{H}$. (b) The field $\boldsymbol{H}$ causes $\boldsymbol{S}$ to rotate with the Larmor frequency $\omega_{L}$. The angle between $\boldsymbol{H}$ and $\boldsymbol{S}$ is $\vartheta$, and $\chi(t)$ is the in-plane rotation angle of $\boldsymbol{S}$. The first- and secondorder tunneling processes are shown in (c) and (d), respectively, in which a spin- $\downarrow$ ( $\uparrow$ ) electron(hole)-like quasiparticle $e \downarrow(h \uparrow)$ absorbs a precession quantum $\omega_{L}$ and undergoes a spin flip. Shapiro-like resonances between the Larmor and the Josephson frequencies lead to spin supercurrents that are transferred, e.g., by the process shown in (e) in which two Cooper pairs at energies differing with $2 \mathrm{eV}$ are transferred while one of the quasiparticles in each of the pairs undergoes a spin flip. (f) The Shapiro spin currents may lead to subnanosecond reversal of the precession axis.

the tunnel-limit regime, and explanations of the origins of the spin-polarized Shapiro steps, respectively.

\section{MODEL}

The junction is described by the Hamiltonian $\mathcal{H}=\mathcal{H}_{\text {leads }}+$ $\mathcal{H}_{B}+\mathcal{H}_{T}$. The left $(L)$ and right $(R)$ leads are assumed to consist of $s$-wave superconductors in the clean limit and are described by the BCS Hamiltonian,

$$
\begin{aligned}
\mathcal{H}_{\text {leads }}= & \sum_{\substack{k, \sigma \\
\alpha L L, R}} \xi_{\alpha, k} c_{\alpha, k, \sigma}^{\dagger} c_{\alpha, k, \sigma} \\
& +\sum_{\substack{k \\
\alpha=L, R}}\left[\Delta_{\alpha} c_{\alpha, k, \uparrow}^{\dagger} c_{\alpha,-k, \downarrow}^{\dagger}+\text { H.c. }\right],
\end{aligned}
$$

where the dispersion is $\xi_{\alpha, k}=\hbar^{2} k^{2} / 2 m-\mu_{\alpha}$ and $\mu_{\alpha}$ is the chemical potential of lead $\alpha \in(L, R)$. The superconducting order parameter $\Delta_{\alpha}=\Delta(T) e^{i \varphi_{\alpha}}$ depends in principle on the temperature $T$, but here we restrict the analysis to the zero-temperature case. The superconducting phase difference $\varphi(t)$ is taken to be placed symmetrically across the junction, i.e., $\varphi_{L, R}= \pm \varphi / 2$ and increases linearly in time according to $\varphi(t)=\varphi_{0}+\omega_{J} t$, where $\varphi_{0}$ is the initial phase difference and $\omega_{J}=2 \mathrm{eV} / \hbar$ is the Josephson frequency.

The nanomagnet's spin $S$ is assumed to be large enough to be treated as a classical entity. Under FMR conditions, external $\mathrm{dc}$ and $\mathrm{rf}$ fields are applied to create an effective magnetic field where $V_{k \sigma ; k^{\prime} \sigma^{\prime}}=\left\{V_{0} \delta_{\sigma \sigma^{\prime}}+V_{S}[\boldsymbol{S}(t) \cdot \boldsymbol{\sigma}]\right\} \delta\left(k-k^{\prime}\right)$ and $\boldsymbol{\sigma}=$ $\left(\sigma_{x}, \sigma_{y}, \sigma_{z}\right)$ are the Pauli matrices. This Hamiltonian contains spin-conserving hopping amplitudes $V_{0}+V_{S} S \cos \vartheta \sigma_{z}$ as well as spin-flip hopping amplitudes $V_{S} S \sin \vartheta e^{-i \omega_{L} t \sigma_{z}} \sigma_{x}$.

Our approach used for calculating the transport properties, which is presented in more detail in Appendix A, involves using nonequilibrium Green's-function techniques in which the Green's functions describing the leads are given by their quasiclassical approximations and the hopping elements by their Fermi-surface limits $V_{0(S)} \rightarrow v_{0(S)}$. The lead Green's functions are coupled via a $t$-matrix equation that describes the perturbation to the hopping elements. The lead Green's functions determine the currents, which include harmonics related to both the Josephson and the Larmor frequencies and can be expressed as

$$
j_{\alpha}^{\mu}(t)=\sum_{n, m} \exp \left[-i\left(n \varphi_{0}+m \chi_{0}\right)-i\left(n \omega_{J}+m \omega_{L}\right) t\right]\left(j_{\alpha}^{\mu}\right)_{n}^{m},
$$

where $\mu=0$ denotes charge currents and $\mu=1-3$ denote the spin-polarization directions $x, y$, and $z$. The current components $\left(j_{\alpha}^{\mu}\right)_{n}^{m}$ are given in Appendix A. The values of $m$ are restricted due to the precession of spin $S$; a spin flip from spin- $\downarrow$ to spin- $\uparrow$ (spin- $\uparrow$ to spin- $\downarrow$ ) corresponds to absorption (emission) of a precession quantum $\omega_{L}$. Since the tunneling quasiparticles are spin- $1 / 2$ particles, only a single precession quantum can be absorbed or emitted. Therefore, only the values $m=0, \pm 1$ are allowed. Moreover, we define the

TABLE I. Types of currents and the existence of corresponding $\mathrm{dc}$ and ac components. The ac column shows with which harmonics the current oscillates.

\begin{tabular}{lcc}
\hline \hline Type of current & $\mathrm{dc}$ & $\mathrm{ac}$ \\
\hline Charge current & $\checkmark$ & $\omega_{J}$ \\
In-plane spin current off-resonance & $\boldsymbol{X}$ & $\omega_{L}, \omega_{J}$ \\
z-polarized spin current & $\checkmark$ & $\omega_{J}$ \\
In-plane spin current at Shapiro resonance & $\checkmark$ & $\omega_{L}, \omega_{J}$ \\
\hline \hline
\end{tabular}


(a) $\mathrm{D}_{\uparrow}=0.8$
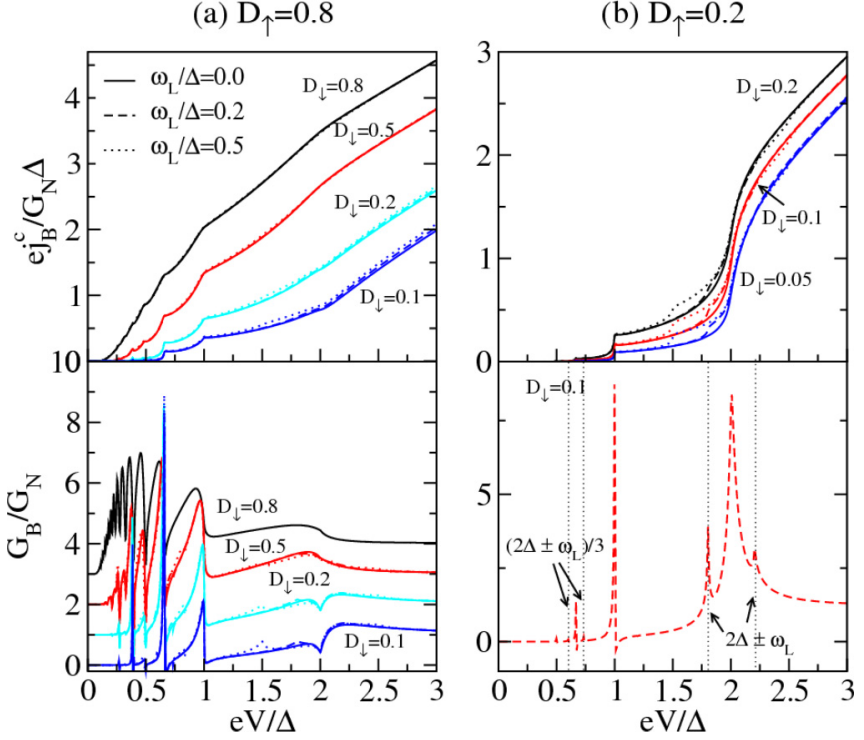

FIG. 2. (Color online) Charge background current and conductance $G_{B}=\partial j_{B}^{c} / \partial V$ for a $\pi$ junction with (a) $\mathcal{D}_{\uparrow}=0.8$ and (b) $\mathcal{D}_{\uparrow}=0.2$. The current is normalized by the normal conductance given by the two spin channels $G_{N}=\left[e^{2} / h\right]\left[\mathcal{D}_{\uparrow}+\mathcal{D}_{\downarrow}\right]$. The conductance curves in panel (a) have been offset with $3\left(\mathcal{D}_{\downarrow}=0.8\right), 2\left(\mathcal{D}_{\downarrow}=0.5\right)$, and $1\left(\mathcal{D}_{\downarrow}=0.2\right)$. In panel (b), the conductance is plotted for $\mathcal{D}_{\uparrow}=0.2, \mathcal{D}_{\downarrow}=0.1$, and $\omega_{L} / \Delta=0.2$. In all plots, $\vartheta=\pi / 8$.

spin-transmission probabilities $\mathcal{D}_{\uparrow(\downarrow)}$ as $\mathcal{D}_{\uparrow(\downarrow)}=4 v_{\uparrow(\downarrow)}^{2} /[1+$ $\left.v_{\uparrow(\downarrow)}^{2}\right]^{2}$, where $v_{\uparrow / \downarrow}=v_{0} \pm v_{S} \cos \vartheta$. Note, however, that a set of $\mathcal{D}_{\uparrow}$ and $\mathcal{D}_{\downarrow}$ values does not uniquely define the hopping elements $v_{0}$ and $v_{S}$ due to the square of $v_{\uparrow(\downarrow)}$ in the definition above. On the other hand, if the voltage bias is replaced by a static phase bias, the junction is in a $\pi(0)$ state if $v_{S}>v_{0}\left(v_{S}<v_{0}\right)[32,40]$, and this additional information combined with the spin-transmission probabilities determines the hopping amplitudes.

\section{CHARGE CURRENTS}

Clearly, the currents in Eq. (3) can be divided into timeindependent as well as time-dependent parts (see Table I for an overview). For an arbitrary bias voltage, the dc currents are in general given by contributions from the $\left(j^{\mu}\right)_{0}^{0}$ components. This kind of current will here be called charge and spin background currents in the spirit of Ref. [41]. At bias voltages fulfilling the Shapiro resonance condition, additional dc components are present. We will start by describing the charge background current. Next, the effects of the spin current will be discussed as a starting point for the analysis of the Shapiro resonances, which then concludes the paper.

Figure 2 shows the current-voltage characteristics as well as the conductance for the charge background current $j_{B}^{c}=$ $\left(j^{\mu=0}\right)_{n=0}^{m=0}$ (from here on, we drop the index $\alpha$ and consider currents on the left side of the interface unless otherwise stated). The value $\omega_{L}=0$ reproduces the usual current steps and conductance peaks at $e V=2 \Delta / n$ due to MAR processes of order $n=1,2, \ldots$ [42-45]. For $\omega_{L} \neq 0$, additional features appear at voltages $e V=\left(2 \Delta \pm \omega_{L}\right) / n$, where $n=1,3, \ldots$ due to the emission or absorption of precession quanta. This structure can clearly be seen in the tunnel-limit conductance where it is displayed as distinct satellite peaks [Fig. 2(b)]. The subharmonic gap structure is similar to that of the current-voltage characteristics generated by photon-assisted MAR, which shows features in the form of satellite peaks due to absorption or emission of one or more photons [41]. However, due to the restriction of only a single emitted or absorbed precession quantum in the spin-precession case, there is only one satellite peak on each side of the feature corresponding to a MAR process of order $n$ [see Figs. 1(c) and 1(d) for processes with $n=1$ and 2]. Additionally, for
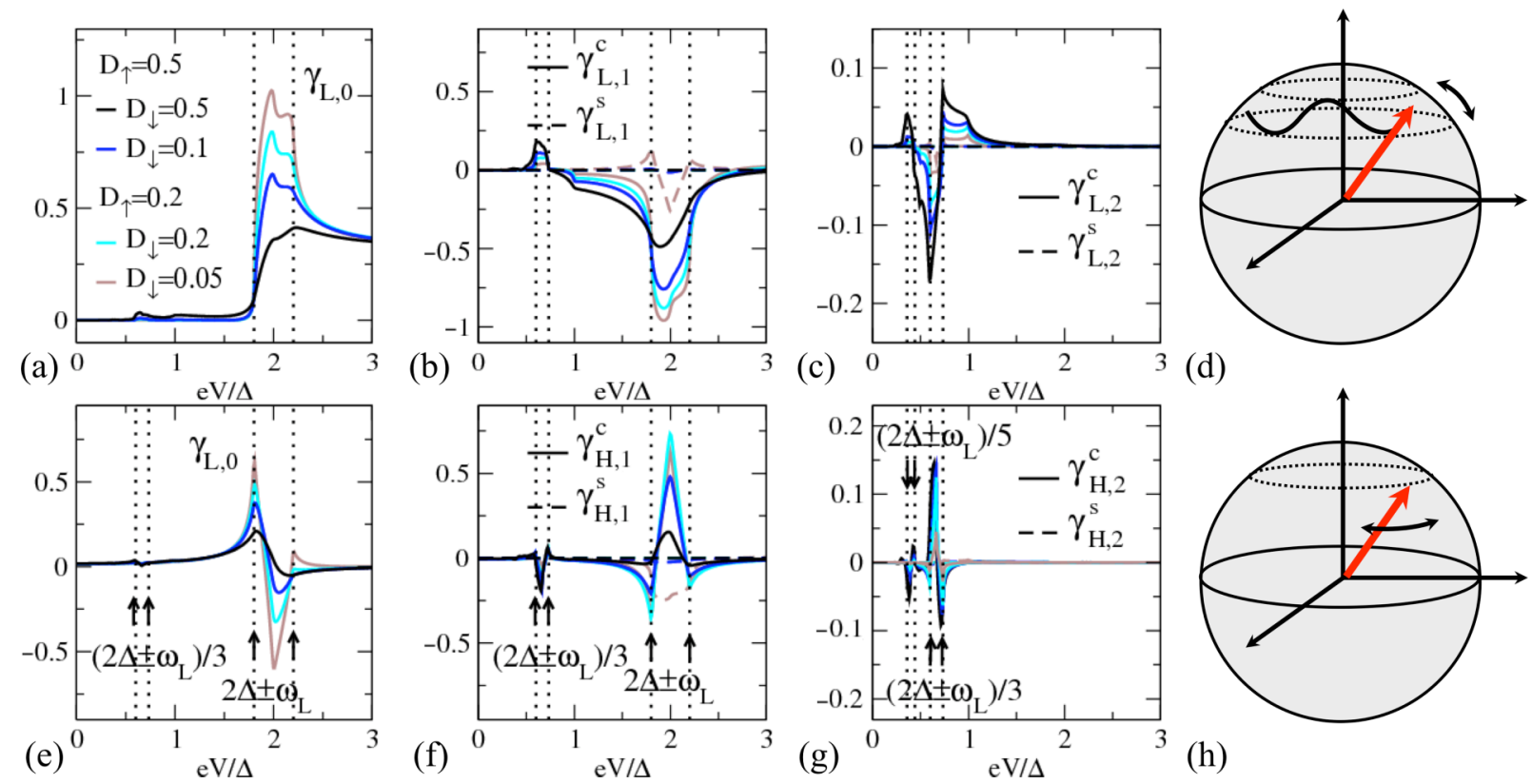

(d)

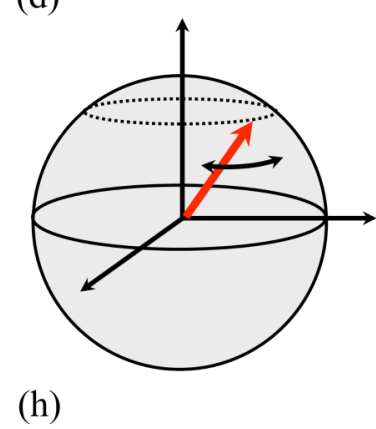

FIG. 3. (Color online) Background torque components as functions of bias voltage for a $\pi$ junction. (a)-(c) Dampinglike components describing spin nutations (d). (e)-(g) Fieldlike components describing oscillations of the precession frequency (h). In all panels, $\vartheta=\pi / 8$. 
a MAR process of order $n$ that includes an exchange of energy $\omega_{L}$, there are $n$ possible paths [Fig. 1(d) shows the two possible absorption paths for $n=2]$. The energy exchange causes a spin flip that introduces a minus sign in a subsequent Andreev reflection amplitude due to the change between the spinors $\left(\psi_{\uparrow}, \psi_{\downarrow}^{\dagger}\right)^{T} \leftrightarrow\left(\psi_{\downarrow}, \psi_{\uparrow}^{\dagger}\right)^{T}$. As a result, the total Andreev reflection is suppressed due to destructive interference in processes where $n=2,4, \ldots$ but is enhanced in processes for which $n$ is an odd number. Consequently, only the MAR processes with $n=1,3, \ldots$ display side peaks.

\section{TORQUES AND SHAPIRO RESONANCES}

Now, we turn to discuss the spin current and its effects. The spin current generates a spin-transfer torque on $S$ given by $\boldsymbol{\tau}(t)=\boldsymbol{j}_{L}(t)-\boldsymbol{j}_{R}(t)$. This torque can then be expressed in terms of $\boldsymbol{S}$ as

$$
\boldsymbol{\tau}(t)=\frac{\gamma_{H}(t)}{S} \gamma \boldsymbol{H} \times \boldsymbol{S}+\frac{\gamma_{L}(t)}{S^{2}} \dot{\boldsymbol{S}} \times \boldsymbol{S},
$$

where

$$
\begin{aligned}
\gamma_{H / L}(t)= & \gamma_{H / L, 0} \mathcal{D}_{S} c_{H / L, \vartheta} \\
& +\sum_{n=1}^{\infty}\left\{\gamma_{H / L, n}^{c} \mathcal{D}_{S} c_{H / L, \vartheta} \cos \left(n \omega_{J} t\right)\right. \\
& \left.+\gamma_{H / L, n}^{s}\left[\mathcal{D}_{\uparrow}-\mathcal{D}_{\downarrow}\right] c_{H / L, \vartheta}^{-1} \sin \left(n \omega_{J} t\right)\right\},
\end{aligned}
$$

with $c_{H, \vartheta}=\cos \vartheta, c_{L, \vartheta}=1$, and $\mathcal{D}_{S}=4 v_{S}^{2} /\left[1+v_{S}^{2}\right]^{2}$. The $\gamma_{L, 0}$ component describes a steady damping, whereas the higher harmonics $\gamma_{L, n \neq 0}^{c / s}$ describe nutations of the spin [38]. The torque associated with the $\gamma_{H, 0}, \gamma_{H, n}^{c / s}$ components instead acts like a magnetic field that shifts the precession frequency. The lowest-order components are plotted in Fig. 3 where it can be seen that the torque components exhibit the same odd-even effect as the charge current.

For voltages at which the Larmor and Josephson frequencies are commensurate $V_{n}^{m}=-(m / n) \omega_{L} / 2 e$ and $n, m \neq 0$, Shapiro resonances may occur [see the inset of Fig. 4(a)]. These Shapiro currents give a contribution to the dc spin current that has an in-plane polarization, that in turn adds a dc contribution to the spin-transfer torque. In a first-order approximation, the dc torque will tilt the spin away from the $z$ axis so that it precesses around a new $z$ direction. Treating this effect self-consistently, we find that the Shapiro currents may be able to reverse the spin's direction. To investigate the effects of the dc Shapiro torques, the corresponding total torque components $\gamma_{L / H, 1}^{c}$ and $\gamma_{L / H, 2}^{c}$ are plotted in Fig. 4 at voltages $e V=\omega_{L} / 2$ and $\omega_{L} / 4$, respectively. $\gamma_{H, 1 / 2}^{c}$ includes the Shapiro contribution, which should hence be enhanced compared to $\gamma_{L, 1 / 2}^{c}$ in order to reduce the spin-reversal times. The other torque components are close to zero and therefore are not shown. As can be seen in Fig. 4(a), the Shapiro torques strongly depend on the junction transparency. The Shapiro torques also display an oscillatory behavior as a function of $\omega_{L}$ as shown in the left panel of Fig. 4(b). The right panel of Fig. 4(b) shows that it is sufficient to choose small precession angles for the spin. The existence of a dc current that is spin polarized in the $x y$ plane implies that the rotational symmetry around the $z$ axis is broken and that the magnitude of the
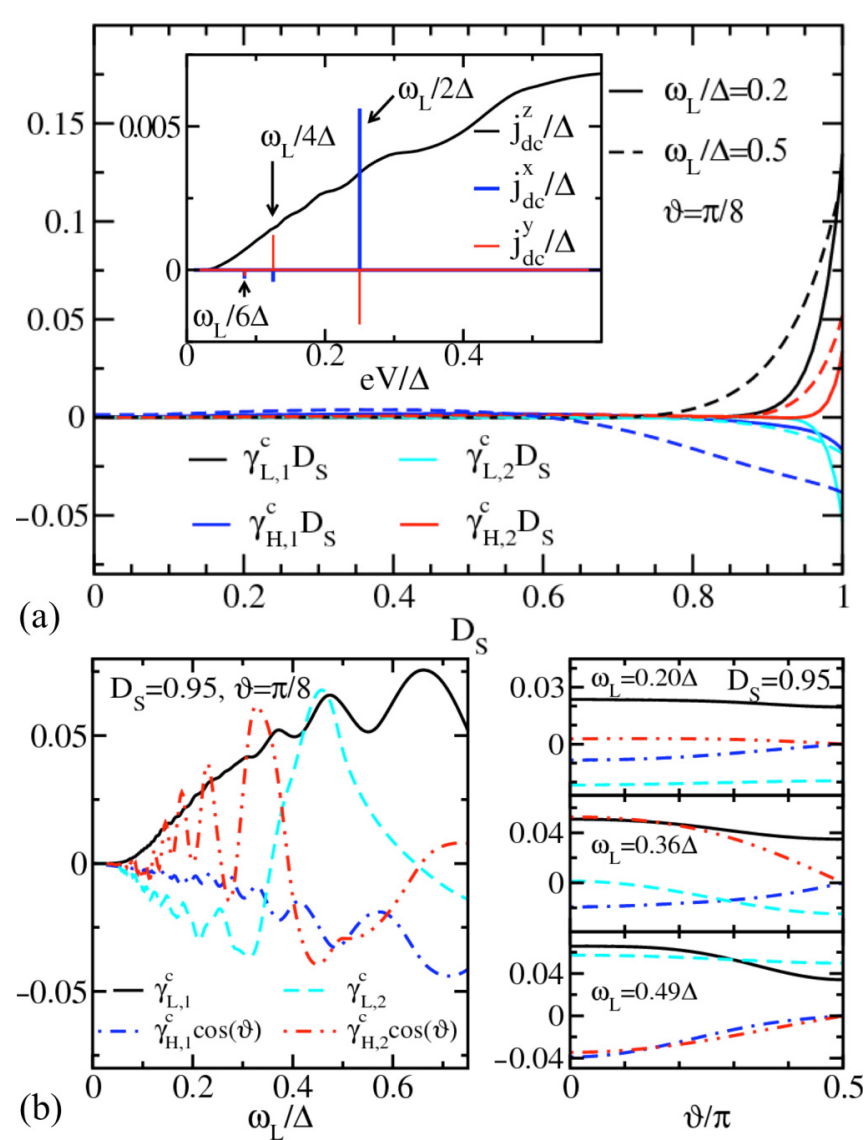

FIG. 4. (Color online) (a) Components of the total torque evaluated at the Shapiro resonance voltages $\gamma_{L / H, 1}^{c}\left(e V=\omega_{L} / 2 \Delta\right)$ and $\gamma_{L / H, 2}^{c}\left(e V=\omega_{L} / 4 \Delta\right)$ as a function of $\mathcal{D}_{S}$ for a junction with $v_{0}=0$. The Shapiro steps depend strongly on $\mathcal{D}_{S}$ and are finite only for high transparencies. Inset: Total dc spin current as a function of bias voltage for $\omega_{L}=0.5 \Delta, \vartheta=\pi / 8$, and $\mathcal{D}_{S}=0.95$. The in-plane spin current exhibits peaks due to Shapiro resonances at the resonant voltages $e V=\omega_{L} / 2 n$. (b) The torque components as functions of $\omega_{L}$ (left panel) and precession angle $\vartheta$ (right panels) showing that $\gamma_{L / H, 2}^{c}$ is sensitive to the precession frequency and that $\gamma_{L / H, 1 / 2}^{c}$ is large even for small precession angles. In all panels, $\varphi_{0}=0$ and $\chi_{0}=0$.

Shapiro currents depends on the initial angle of nanomagnet's magnetization direction $\chi_{0}$. This behavior is analogous to the dependence on the initial phase difference $\varphi_{0}$, which also is present in the transport characteristics of microwave-irradiated Josephson junctions [41].

Realistic values of $\omega_{L}$ may be smaller than the ones shown in Fig. 2, which were chosen for clarity. Consider an SQPC consisting of $\mathrm{Nb}$, whose superconducting gap is $\Delta \sim 1 \mathrm{meV}$, that contains a nanomagnet which under FMR conditions reaches precession frequencies of $\omega_{L} \sim 10 \mathrm{GHz}$, which is well below the critical magnetic field $H_{c}$ of $\mathrm{Nb}$ [22]. Then, typically $\omega_{L} / \Delta=0.01$, and the temperature is restricted to $T<100 \mathrm{mK}$. Increasing $\boldsymbol{H}$ closer to the critical-field value decreases $\Delta$ and hence increases the ratio $\omega_{L} / \Delta$, which allows for a better resolution of the subgap structures of $j_{B}^{c}$. Alternatively, using the ac Josephson current to resonantly excite the FMR mode of $\boldsymbol{S}(t), \omega_{L}$ values corresponding to an effective magnetic field $|\boldsymbol{H}|>H_{c}$ can be achieved [26-28]. Detection of the spin-polarized Shapiro currents would then 
be a measurement of the FMR frequency of the nanomagnet. To estimate the effectiveness of the Shapiro resonances for spin reversal, self-consistent calculations were performed in which the spin dynamics were determined using the calculated spin-transfer torques. A spin $S \sim 50 \hbar$, a contact width of $\sim 100 \mathrm{~nm}$ giving the number of conduction channels $n_{\mathrm{c}} \sim$ 600 , and $\omega_{L}=10 \mathrm{GHz}$ give an estimated switching time of $\sim 0.1 \mathrm{~ns}$.

\section{CONCLUSIONS}

In conclusion, we have calculated the charge and spin currents through a voltage-biased superconducting point contact coupled to the magnetization dynamics of a nanomagnet. We have shown that coherent multiple Andreev reflection in the presence of the Larmor dynamics leads to a subharmonic gap structure of the dc charge and spin currents with features at $e V=\left(2 \Delta \pm \omega_{L}\right) / n$, where $n$ is an odd number due to interference. In addition, resonance between the Josephson and the Larmor frequencies leads to a rectification of the spinpolarized supercurrent and the generation of spin-polarized Shapiro steps, which is in contrast to the current through a weak link coupled to a nanomagnet whose magnetization performs a precessional motion. In that case, the resulting time-dependent magnetic field produces Shapiro-type resonances that give contributions to the charge current [46]. Estimates show that the spin-polarized Shapiro steps, that depend sensitively on the bias voltage, may lead to subnanosecond reversal of the magnetization direction.

\section{ACKNOWLEDGMENTS}

We wish to thank J. C. Cuevas for valuable discussions. C.H. and W.B. were supported by the DFG and SFB 767. M.F. acknowledges support from the Swedish Research Council (VR).

\section{APPENDIX A: DETAILS OF THE APPROACH}

The transport properties are obtained using nonequilibrium Green's-function techniques through the Hamiltonian approach $[13,45,47]$ in which the leads are described by KeldyshGreen's functions in the quasiclassical approximation [48-50]. The nanomagnet creates a spin-active interface that can be treated as a strong impurity [51]. The resulting boundary condition can be solved using a $t$-matrix equation expressed in terms of the tunneling amplitudes and the quasiclassical Green's functions.

A quasiclassical Green's function, or propagator, describes quasiparticles at the Fermi surface with momentum $\boldsymbol{p}_{F}$ moving along classical trajectories with velocity $\boldsymbol{v}_{F}\left(\boldsymbol{p}_{F}\right)$. These Green's functions can be written in the form

$$
\check{g}\left(\boldsymbol{p}_{F}, \boldsymbol{R} ; \varepsilon, t\right)=\left(\begin{array}{cc}
\hat{g}^{R}\left(\boldsymbol{p}_{F}, \boldsymbol{R} ; \varepsilon, t\right) & \hat{g}^{K}\left(\boldsymbol{p}_{F}, \boldsymbol{R} ; \varepsilon, t\right) \\
0 & \hat{g}^{A}\left(\boldsymbol{p}_{F}, \boldsymbol{R} ; \varepsilon, t\right)
\end{array}\right),
$$

where " " " denotes a matrix in Keldysh space and the Green's functions $\hat{g}^{R, K, A}$ are the retarded $(R)$, Keldysh $(K)$, and advanced $(A)$ Green's functions in Nambu space, that is denoted by “^." The Green's functions obey the Eilenberger equation [50],

$$
i \hbar v_{F} \cdot \nabla \check{g}+\left[\varepsilon \hat{\tau}_{3}-\check{\mathcal{H}}, \check{g}\right]_{\circ}=0,
$$

that is complemented by the normalization condition $\check{g} \circ \check{g}=$ $-\pi^{2} \breve{1}$. The symbol "o" denotes matrix multiplication and convolution over common time arguments.

A first step in calculating the transport properties of the junction is to solve Eq. (A2) separately in each lead under the assumption that the tunneling is switched off. In equilibrium, the solutions are given by the Green's functions

$$
\hat{g}_{\alpha}^{R, A}(\varepsilon)=\hat{\tau}_{3} g^{R, A}(\varepsilon)+\hat{\tau}_{1} f^{R, A}(\varepsilon) i \sigma_{y}
$$

and

$$
\hat{g}_{\alpha}^{K}(\varepsilon)=\left[\hat{g}_{\alpha}^{R}(\varepsilon)-\hat{g}_{\alpha}^{A}(\varepsilon)\right] \tanh \left(\frac{\varepsilon}{2 T}\right)
$$

where

$$
g^{R, A}(\varepsilon)=-\pi \varepsilon / \Omega^{R, A}, \quad f^{R, A}(\varepsilon)=-\pi \Delta / \Omega^{R, A},
$$

and

$$
\Omega^{R, A}=\sqrt{|\Delta|^{2}-(\varepsilon \pm i 0)^{2}} .
$$

The effects of $\varphi(t)$ are accounted for by a transformation $\hat{\mathcal{U}}_{\alpha}=\exp \left[i \varphi_{\alpha}(t) \hat{\tau}_{3} / 2\right]$ of the lead Green's functions. The voltage-biased lead Green's functions $\check{g}_{\alpha}\left(t, t^{\prime}\right)$ are then expressed in terms of the equilibrium ones $\check{g}_{\alpha}\left(t-t^{\prime}\right)=$ $\int(d \varepsilon / 2 \pi) e^{-i \varepsilon\left(t-t^{\prime}\right)} \check{g}_{\alpha}(\varepsilon)$ as $\check{g}_{\alpha}\left(t, t^{\prime}\right)=\hat{\mathcal{U}}_{\alpha}^{\dagger}(t) \check{g}_{\alpha}\left(t-t^{\prime}\right) \hat{\mathcal{U}}_{\alpha}\left(t^{\prime}\right)$.

Next, the quasiclassical trajectories in the left and right leads are connected via the coupling described by the tunnel Hamiltonian Eq. (2). Within the quasiclassical approximation, the hopping amplitudes are replaced by their Fermi-surface counterparts, which here means that $V_{0}$ and $V_{S}$ are replaced by $v_{0}=\pi N_{F} V_{0}$ and $v_{S}=\pi N_{F} S V_{S}$, where $N_{F}$ is the normal density of states at the Fermi energy. The resulting hopping matrix in Nambu space is then

$$
\hat{v}(t)=\left(\begin{array}{cc}
v_{0}+v_{S} \boldsymbol{e}_{S}(t) \cdot \boldsymbol{\sigma} & 0 \\
0 & v_{0}-v_{S} \sigma_{y}\left[\boldsymbol{e}_{S}(t) \cdot \boldsymbol{\sigma}\right] \sigma_{y}
\end{array}\right),
$$

where $\boldsymbol{e}_{S}$ is the unit vector that describes the precessional motion of spin $\boldsymbol{S}$ defined in Sec. II. The coupling between the left and the right leads is captured by the so-called $t$-matrix equation that describes a perturbation to the hopping element and is given by

$$
\begin{aligned}
\check{t}_{\alpha}\left(t, t^{\prime}\right)= & \check{\Gamma}_{\alpha}\left(t, t^{\prime}\right) \\
& +\int d t_{1} \int d t_{2} \check{\Gamma}_{\alpha}\left(t, t_{1}\right) \check{g}_{\alpha}\left(t_{1}, t_{2}\right) \check{t}_{\alpha}\left(t_{2}, t^{\prime}\right),
\end{aligned}
$$

where $\check{\Gamma}_{L / R}\left(t, t^{\prime}\right)=\breve{v}(t) \check{g}_{R / L}\left(t, t^{\prime}\right) \check{v}\left(t^{\prime}\right)$. The time dependence of the superconducting phase of the Green's functions is transferred to the hopping elements by the transformation $\hat{\mathcal{U}}_{\alpha}(t)$ according to $\hat{v}_{L R / R L}(t)=\hat{\mathcal{U}}_{L / R}(t) \hat{v}(t) \hat{\mathcal{U}}_{R / L}^{\dagger}(t)=$ $\exp \left[ \pm i \varphi(t) \hat{\tau}_{3} / 2\right] \hat{v}(t)$. The $t$ matrices can be shown to depend on energies containing multiples of frequencies $\omega_{J}$ and $\omega_{L}$. Explicitly, the $t$ matrices have a time dependence of the form

$$
\begin{aligned}
\check{t}_{\alpha}\left(t, t^{\prime}\right)= & \sum_{n, m} \exp \left[-i\left(n \varphi_{0}+m \chi_{0}\right)\right. \\
& \left.+i\left(n \omega_{J}+m \omega_{L}\right) t^{\prime}\right]\left[\check{t}_{\alpha}\left(t-t^{\prime}\right)\right]_{n}^{m},
\end{aligned}
$$


where the components are given by

$$
\left[\check{t}_{\alpha}(t)\right]_{n}^{m}=\int \frac{d \varepsilon}{2 \pi} e^{-i \varepsilon t}\left[\check{t}_{\alpha}\left(\varepsilon_{n}^{m}\right)\right],
$$

and $\varepsilon_{n}^{m}=\varepsilon+n \omega_{J}+m \omega_{L}$

From now on, we drop the index $\alpha$ and show how to calculate the $t$ matrices on the left side of the junction. Defining $\check{t}_{n}^{m}=\left[\check{t}\left(\varepsilon_{n}^{m}\right)\right]$ and $\check{g}_{n}^{m}=\check{g}\left(\varepsilon_{n}^{m}\right)$ and Fourier transforming the $t$-matrix equation, one obtains the algebraic equation,

$$
\check{t}_{n}^{m}=\check{\Gamma}_{n}^{m}+\sum_{l}\left\{\check{A}_{n, n}^{m, l} \check{\check{t}}_{n}^{l}+\check{B}_{n, n+1}^{m, l} \check{t}_{n+1}^{l}+\check{B}_{n, n-1}^{m, l} \check{t}_{n-1}^{l}\right\},
$$

where the retarded components are given by

$$
\begin{aligned}
& {\left[\hat{\Gamma}^{R}\right]_{n}^{m}=\sum_{j}\left(\begin{array}{cc}
v^{m-j} v^{j}\left[g^{R}\right]_{-(1 / 2)}^{j} \delta_{n, 0} & e^{-i \varphi_{0}} v^{m-j} i \sigma_{y}\left(v^{j}\right)^{\dagger}\left[f^{R}\right]_{1 / 2}^{j} \delta_{n, 1} \\
e^{i \varphi_{0}}\left(v^{m-j}\right)^{\dagger} i \sigma_{y} v^{j}\left[f^{R}\right]_{-(1 / 2)}^{j} \delta_{n,-1} & -\left(v^{m-j}\right)^{\dagger}\left(v^{j}\right)^{\dagger}\left[g^{R}\right]_{1 / 2}^{j} \delta_{n, 0}
\end{array}\right),} \\
& {\left[\hat{A}^{R}\right]_{n, n}^{m, l}=\sum_{j=-2}^{2}\left(\begin{array}{cc}
v^{m-j} v^{j-l}\left[g^{R}\right]_{n-1 / 2}^{j}\left[g^{R}\right]_{n}^{l} & v^{m-j} v^{j-l} i \sigma_{y}\left[g^{R}\right]_{n-1 / 2}^{j}\left[f^{R}\right]_{n}^{l} \\
-\left(v^{m-j}\right)^{\dagger}\left(v^{j-l}\right)^{\dagger} i \sigma_{y}\left[g^{R}\right]_{n+1 / 2}^{j}\left[f^{R}\right]_{n}^{l} & \left(v^{m-j}\right)^{\dagger}\left(v^{j-l}\right)^{\dagger}\left[g^{R}\right]_{n+1 / 2}^{j}\left[g^{R}\right]_{n}^{l}
\end{array}\right),} \\
& {\left[\hat{B}^{R}\right]_{n, n+1}^{m, l}=\sum_{j=-2}^{2} e^{i \varphi_{0}}\left(v^{m-j}\right)^{\dagger} i \sigma_{y} v^{j-l}\left[f^{R}\right]_{n+1 / 2}^{j}\left(\begin{array}{cc}
0 & 0 \\
{\left[g^{R}\right]_{n+1}^{l}} & {\left[f^{R}\right]_{n+1}^{l} i \sigma_{y}}
\end{array}\right),}
\end{aligned}
$$

and

$$
\left[\hat{B}^{R}\right]_{n, n-1}^{m, l}=\sum_{j=-2}^{2} e^{-i \varphi_{0}} \nu^{m-j} i \sigma_{y}\left(v^{j-l}\right)^{\dagger}\left[f^{R}\right]_{n-1 / 2}^{j}\left(\begin{array}{cc}
i \sigma_{y}\left[f^{R}\right]_{n-1}^{l} & -\left[g^{R}\right]_{n-1}^{l} \\
0 & 0
\end{array}\right) .
$$

Here, we have defined $v^{0}=v_{0}+v_{S} \sigma_{z} \cos \vartheta, v^{ \pm 1}=\frac{v_{S}}{2}\left[\sigma_{x} \pm i \sigma_{y}\right] \sin \vartheta$, and $v^{j}=0$ if $|j| \geqslant 2$. The advanced and Keldysh matrices are similarly defined. Collecting the terms as

$$
\begin{aligned}
\bar{A}_{n, n} & =\left(\begin{array}{ccc}
\check{A}_{n, n}^{1,1} & \check{A}_{n, n}^{1,0} & \check{0}^{\prime} \\
\check{A}_{n, n}^{0,1} & \check{A}_{n, n}^{0,0} & \check{A}_{n, n}^{0,-1} \\
\check{0} & \check{A}_{n, n}^{-1,0} & \check{A}_{n, n}^{-1,-1}
\end{array}\right), \quad \bar{B}_{n, n \pm 1}=\left(\begin{array}{ccc}
\check{B}_{n, n \pm 1}^{1,1} & \check{B}_{n, n \pm 1}^{1,0} & \multicolumn{0}{c}{} \\
\check{B}_{n, n \pm 1}^{0,1} & \check{B}_{n, n \pm 1}^{0,0} & \check{B}_{n, n \pm 1}^{0,-1} \\
\check{0} & \check{B}_{n, n \pm 1}^{-1,0} & \check{B}_{n, n \pm 1}^{-1,-1}
\end{array}\right), \\
\bar{t}_{n} & =\left(\begin{array}{c}
\check{t}_{n}^{1} \\
\check{t}_{n}^{0} \\
\check{t}_{n}^{-1}
\end{array}\right), \quad \bar{\Gamma}_{n}^{1}=\left(\begin{array}{c}
\check{\Gamma}_{n}^{0} \\
\check{\Gamma}_{n}^{-1}
\end{array}\right),
\end{aligned}
$$

the $t$-matrix equation becomes

$$
\bar{t}_{n}=\bar{\Gamma}_{1} \delta_{n, 1}+\bar{\Gamma}_{0} \delta_{n, 0}+\bar{\Gamma}_{-1} \delta_{n,-1}+\bar{B}_{n, n+1} \bar{t}_{n+1}+\bar{A}_{n, n} \bar{t}_{n}+\bar{B}_{n, n-1} \bar{t}_{n-1} .
$$

A recursive solution to Eq. (A15) can be found by defining the auxiliary matrices,

$$
\begin{aligned}
\bar{X}_{n, n+1} & =\left[\overline{1}-\bar{A}_{n, n}-\bar{B}_{n, n+1} \bar{X}_{n+1, n+2}\right]^{-1} \bar{B}_{n, n-1} \quad \text { for } n \leqslant N, \\
\bar{X}_{n, n+1} & =\overline{0} \quad \text { for } n>N, \\
\bar{X}_{n, n-1} & =\left[\overline{1}-\bar{A}_{-n,-n}-\bar{B}_{-n,-n-1} \bar{X}_{-n-1,-n-2}\right]^{-1} \bar{B}_{-n,-n+1} \quad \text { for }-n \geqslant-N, \\
\bar{X}_{-n,-n-1} & =\overline{0} \quad \text { for }-n<-N,
\end{aligned}
$$

which results in the initial condition,

$$
\left(\begin{array}{ccc}
{\left[\overline{1}-\bar{A}_{1,1}-\bar{B}_{1,2} \bar{X}_{2,3}\right]} & -\bar{B}_{1,0} & \overline{0} \\
-\bar{B}_{0,1} & {\left[\overline{1}-\bar{A}_{0,0}\right]} & -\bar{B}_{0,-1} \\
\overline{0} & -\bar{B}_{-1,0} & {\left[\overline{1}-\bar{A}_{-1,-1}-\bar{B}_{-1,-2} \bar{X}_{-2,-3}\right]}
\end{array}\right)\left(\begin{array}{c}
\bar{t}_{1} \\
\bar{t}_{0} \\
\bar{t}_{-1}
\end{array}\right)=\left(\begin{array}{c}
\bar{\Gamma}_{1} \\
\bar{\Gamma}_{0} \\
\bar{\Gamma}_{-1}
\end{array}\right) .
$$

The $t$ matrices can then be obtained recursively from the auxiliary matrices according to

$$
\begin{gathered}
\bar{t}_{n}=\bar{X}_{n, n+1} \bar{t}_{n-1}, \\
\bar{t}_{-n}=\bar{X}_{-n,-n-1} \bar{t}_{-n+1} .
\end{gathered}
$$

These $t$ matrices are then used to calculate the perturbed lead Green's functions and subsequently the charge and spin currents through the junction.

The calculation of the currents starts by noting that the lead Green's functions can be divided into two categories depending on if the quasiparticles they describe have trajectories leading 
towards or away from the interface. These Green's functions are called incoming and outgoing propagators $\check{g}^{i}$ and $\check{g}^{o}$, respectively, and are given by [52]

$$
\check{g}_{\alpha}^{i, o}\left(t, t^{\prime}\right)=\check{g}_{\alpha}\left(t, t^{\prime}\right)+\left[\left(\check{g}_{\alpha} \pm i \pi \check{1}\right) \circ \check{t}_{\alpha} \circ\left(\check{g}_{\alpha} \mp i \pi \check{1}\right)\right]\left(t, t^{\prime}\right) .
$$

The charge and spin currents can then be calculated as the difference between these propagators according to

$$
j_{\alpha}^{\mu}=\int \frac{d \varepsilon}{8 \pi i} \operatorname{Tr}\left\{\hat{\kappa}^{\mu}\left[\hat{g}_{\alpha}^{i,<}(\varepsilon, t)-\hat{g}_{\alpha}^{o,<}(\varepsilon, t)\right]\right\},
$$

where we have defined $\hat{\kappa}^{0}=e \hat{\tau}_{3}$ for the charge current and $\hat{\kappa}^{i}=\operatorname{diag}\left(\sigma_{i}, \sigma_{y} \sigma_{i} \sigma_{y}\right) / 2$ for a spin current with a polarization in the $i=x, y, z$ direction. With the help of Eq. (A20), the currents can then be obtained as

$$
j_{\alpha}^{\mu}=\int \frac{d \varepsilon}{4} \operatorname{Tr}\left\{\hat{\kappa}^{\mu}\left[\check{t}_{\alpha}, \check{g}_{\alpha}\right]_{\circ}^{<}\right\},
$$

which, with the help of Eqs. (A7) and (A8), can be written as

$$
j_{\alpha}^{\mu}(t)=\sum_{n, m} \exp \left[-i\left(n \varphi_{0}+m \chi_{0}\right)-i\left(n \omega_{J}+m \omega_{L}\right) t\right]\left(j_{\alpha}^{\mu}\right)_{n}^{m},
$$

where

$$
\left(j_{\alpha}^{\mu}\right)_{n}^{m}=\int \frac{d \varepsilon}{4} \operatorname{Tr}\left\{\hat{\kappa}^{\mu}\left[\check{t}\left(\varepsilon_{n}^{m}\right) \check{g}\left(\varepsilon_{0}^{0}\right)-\check{g}\left(\varepsilon_{n}^{m}\right) \check{t}\left(\varepsilon_{n}^{m}\right)\right]^{<}\right\}
$$

are the current components of Eq. (3).

\section{APPENDIX B: TUNNEL-LIMIT EXPRESSIONS}

In the tunnel limit $v_{0}, v_{S} \ll 1$, analytical expressions for the charge and spin currents can be obtained. Defining $\delta V^{ \pm}=\left[e V-2 \Delta \pm \omega_{L}\right] / 2 \Delta$, the height of the charge current steps at $\delta V^{ \pm} \ll 1$ generated by the spin precession can be approximated in the tunnel limit by

$$
j_{ \pm}^{c} \approx \Theta\left(\delta V^{ \pm}\right) e \Delta \frac{2}{\pi} v_{s}^{2} \sin ^{2}(\vartheta) I\left(\left[e V \pm \omega_{L}\right] / 2 \Delta\right),
$$

where $\Theta$ is the Heaviside step function, $I(a)=$ $\left[2 a E\left(\sqrt{1-1 / a^{2}}\right)-K\left(\sqrt{1-1 / a^{2}}\right) / a\right]$, and $K$ and $E$ are the complete elliptic integrals of the first and second kinds, respectively. In the limit $\omega_{L} \ll 2 \Delta$, one obtains $I\left(\left[e V \pm \omega_{L}\right] / 2 \Delta\right) \approx$ $\frac{\pi}{2}\left[1+\frac{3}{2} \delta V^{ \pm}\right]$, and the heights of the charge current steps at $V=\left(2 \Delta \pm \omega_{L}\right) / e$ are thus $\propto v_{s}^{2} \sin ^{2} \vartheta$.

The spin background current can be divided into a spinfilter current and a spin-pump current $\boldsymbol{j}_{B}^{s}=\boldsymbol{j}_{\text {filter }}^{s}+\boldsymbol{j}_{\text {pump }}^{s}$ in the tunnel limit. The spin-filter current is given by $\boldsymbol{j}_{\text {filter }}^{s}=$ $\boldsymbol{e}_{z}(\Delta / 2 \pi)\left(\mathcal{D}_{\uparrow}-\mathcal{D}_{\downarrow}\right) I(e V / 2 \Delta)$ for $e V \geqslant 2 \Delta$ and reproduces the results of Ref. [17]. The spin-pump current can correspondingly be approximated by $\boldsymbol{j}_{\text {pump }, \pm}^{s} \approx \pm \boldsymbol{e}_{z} j_{ \pm}^{c} / 2 e$. For $e V>$ $2 \Delta+\omega_{L}$ and $\omega_{L} \ll \Delta$, the total spin-pump current is given by $j_{\text {pump }}^{s}=\boldsymbol{e}_{z}(3 / 4) v_{s}^{2} \sin ^{2}(\vartheta) \omega_{L}$ and is hence proportional to $\omega_{L}$ and does not depend on the bias voltage.

The dc spin-transfer torque components, can, in the tunnel limit, be expressed as

$$
\gamma_{L, 0} \approx-\frac{\Delta}{\pi} \frac{v_{S}^{2}}{\omega_{L}} \operatorname{Re}\left[I_{0}^{\tau}\left(\frac{e V+\omega_{L}}{2 \Delta}\right)-I_{0}^{\tau}\left(\frac{e V-\omega_{L}}{2 \Delta}\right)\right],
$$

and

$$
\begin{aligned}
\gamma_{H, 0} \approx & \frac{\Delta}{2 \pi} \frac{v_{S}^{2}}{\omega_{L}} \cos (\vartheta) \operatorname{Im}\left[I_{0}^{\tau}\left(\frac{e V+\omega_{L}}{2 \Delta}\right)\right. \\
& \left.-2 I_{0}^{\tau}\left(\frac{e V}{2 \Delta}\right)+I_{0}^{\tau}\left(\frac{e V-\omega_{L}}{2 \Delta}\right)\right],
\end{aligned}
$$

where

$$
\begin{aligned}
I_{0}^{\tau}(a)= & 2\left(i\left\{2|a|\left[K\left(\frac{1}{|a|}\right)-E\left(\frac{1}{|a|}\right)\right]-\frac{1}{|a|} K\left(\frac{1}{|a|}\right)\right\}\right. \\
& \left.-\operatorname{sgn}(a)\left\{2|a| E\left(\frac{\sqrt{a^{2}-1}}{|a|}\right)-\frac{1}{|a|} K\left(\frac{\sqrt{a^{2}-1}}{|a|}\right)\right\}\right) .
\end{aligned}
$$

The first-harmonics terms are given by $\gamma_{L / H, 1}=$ $\gamma_{L / H, 1}^{c} \cos \left(\omega_{J} t\right)+\gamma_{L / H, 1}^{s} \sin \left(\omega_{J} t\right)$ where the components of the dampinglike torque are given by

$$
\gamma_{L, 1}^{c} \approx-\frac{\Delta}{\pi \omega_{L}} v_{S}^{2} \operatorname{Re}\left[I_{1}^{\tau}\left(\frac{e V+\omega_{L}}{2 \Delta}\right)-I_{1}^{\tau}\left(\frac{e V-\omega_{L}}{2 \Delta}\right)\right],
$$

$$
\begin{aligned}
\gamma_{L, 1}^{s} \approx & \frac{\Delta}{\pi \omega_{L}} v_{0} v_{S} \cos (\vartheta) \operatorname{Im}\left[I_{1}^{\tau}\left(\frac{e V+\omega_{L}}{2 \Delta}\right)-2 I_{1}^{\tau}\left(\frac{e V}{2 \Delta}\right)\right. \\
& \left.+I_{1}^{\tau}\left(\frac{e V-\omega_{L}}{2 \Delta}\right)\right],
\end{aligned}
$$

where $I_{1}^{\tau}(a)=-2 i K(|a|)$. The components of the fieldlike torque are

$$
\begin{aligned}
\gamma_{H, 1}^{c} \approx & -\frac{\Delta}{\pi \omega_{L}} v_{S}^{2} \cos (\vartheta) \operatorname{Im}\left[I_{1}^{\tau}\left(\frac{e V+\omega_{L}}{2 \Delta}\right)-2 I_{1}^{\tau}\left(\frac{e V}{2 \Delta}\right)\right. \\
& \left.+I_{1}^{\tau}\left(\frac{e V-\omega_{L}}{2 \Delta}\right)\right], \\
\gamma_{H, 1}^{s} \approx & -\frac{\Delta}{\pi \omega_{L}} v_{S} v_{0} \operatorname{Re}\left[I_{1}^{\tau}\left(\frac{e V+\omega_{L}}{2 \Delta}\right)-I_{1}^{\tau}\left(\frac{e V-\omega_{L}}{2 \Delta}\right)\right] .
\end{aligned}
$$

\section{APPENDIX C: THE SPIN POLARIZATION OF THE SHAPIRO CURRENT}

The spin polarization of the Shapiro current is a direct consequence of the symmetries of the properties of the $t$ matrices calculated using Eq. (A15). The spin raising/spin lowering $\check{t}_{n}^{m= \pm 1}$ matrices can be shown to contain $t$ matrices of the form $\hat{t}_{n}^{m= \pm 1, X}=t_{n}^{m= \pm 1, X} \operatorname{diag}\left\{\sigma^{ \pm 1},-\sigma_{y} \sigma^{ \pm 1} \sigma_{y}\right\}$, where $\sigma^{ \pm 1}=\left[\sigma_{x} \pm i \sigma_{y}\right] / 2$ and $X$ stands for one of the retarded $(R)$, advanced $(A)$, or Keldysh $(K)$ Nambu matrices. Then, one immediately obtains the result that the Shapiro current is spin polarized in the $x y$ plane and hence can be written in the form $j_{\text {Shapiro }}^{i}=\sum_{n, m= \pm 1} e^{-i\left(n \varphi_{0}+m \chi_{0}\right)}\left(j^{i}\right)_{n}^{m} \delta\left(V-V_{n}^{m}\right)$, where $i=x, y, z$. Defining $\left(j^{i}\right)_{n} \delta\left(V-V_{n}\right) \equiv \sum_{m= \pm 1}\left[\left(j^{i}\right)_{n}^{m}+\right.$ $\left.\left(j^{i}\right)_{-n}^{m}\right] \delta\left(V-V_{n}^{m}\right)$, where $V_{n}= \pm \omega_{L} / 2 e n$, one can write the $n$th component as $\left(\boldsymbol{j}_{\text {Shapiro }}\right)_{n}=\hat{R}^{\dagger}(\boldsymbol{j})_{n} \hat{R} \delta\left(V-V_{n}\right)$, where $n \geqslant 1$ and the transformation $\hat{R}=\exp \left[\frac{i}{2}\left(-n \varphi_{0} \pm \chi_{0}\right) \hat{\boldsymbol{H}} \cdot \boldsymbol{\sigma}\right]$ rotates the vector $(\boldsymbol{j})_{n}$ around the direction of the external magnetic field $\hat{\boldsymbol{H}}$ through an angle $-n \varphi_{0}+\operatorname{sgn}\left(V_{n}\right) \chi_{0}$. 
The physical explanation for the existence of the spinpolarized Shapiro steps is the following. Consider a phasebiased SQPC coupled to a precessing spin and with a bias voltage $e V=0$. As was shown in Refs. [31,32], the resulting quasiparticle scattering leads to induced superconducting spintriplet correlations in the leads close to the tunnel interface. In general, spin-triplet correlations may be characterized by a complex $\boldsymbol{d}$ vector that is given by $\Delta=\boldsymbol{d} \cdot \boldsymbol{\sigma} i \sigma_{y}$ and points along the direction of zero-spin projection of the Cooper pairs. If one similarly defines $\boldsymbol{d}_{\alpha}$ vectors for the induced spin-triplet correlations in lead $\alpha$ of the phase-biased SQPC, one finds that the $\boldsymbol{d}_{\alpha}$ vectors have a time dependence similar to that of the $t$ matrices described above, viz. $\boldsymbol{d}_{\alpha}(t, V=$ $0)=\boldsymbol{d}_{\alpha}^{z}+\boldsymbol{d}_{\alpha}^{1} e^{-i \omega_{L} t}+\boldsymbol{d}_{\alpha}^{-1} e^{i \omega_{L} t}$, where $\boldsymbol{d}_{\alpha}^{z}$ describes a time independent $z$ component and $\boldsymbol{d}_{\alpha}^{ \pm 1}$ are complex vectors in the $x y$ plane [32]. These spin-triplet correlations are able to support a spin-triplet supercurrent that is correspondingly given by $\boldsymbol{j}_{\alpha}(t, V=0)=\boldsymbol{j}_{\alpha}^{z}+\boldsymbol{j}_{\alpha}^{1} e^{-i \omega_{L} t}+\boldsymbol{j}_{\alpha}^{-1} e^{i \omega_{L} t}$, where $\boldsymbol{j}_{\alpha}^{z}$ is a $z$ component and $\boldsymbol{j}_{\alpha}^{ \pm 1}$ contains $x y$ components. Replacement of the phase bias with a voltage bias that is on-resonance leads to a rectification of the higher harmonics of the time-dependent current. Since the higher harmonics in this case are nothing but $\boldsymbol{j}_{\alpha}^{ \pm 1} e^{\mp i \omega_{L} t}$, the Shapiro steps are $x y$ spin polarized. The existence of a dc current that is spin polarized in the $x y$ plane implies that the rotational symmetry around the $z$ axis is broken and that the magnitude of the Shapiro currents depends on the initial angle of the nanomagnet's magnetization direction $\chi_{0}$ analogous to the $\varphi_{0}$ dependence of the Shapiro steps displayed by microwave-irradiated Josephson junctions $[41,53,54]$.
[1] M. Eschrig, Phys. Today 64(1), 43 (2011).

[2] Y. Maeno, H. Hashimoto, K. Yoshida, S. Nishizaki, T. Fujita, J. G. Bednorz, and F. Y. Lichtenberg, Nature (London) 372, 532 (1994).

[3] A. P. Mackenzie and Y. Maeno, Rev. Mod. Phys. 75, 657 (2003).

[4] M. Sigrist and K. Ueda, Rev. Mod. Phys. 63, 239 (1991).

[5] B. Kastening, D. K. Morr, D. Manske, and K. Bennemann, Phys. Rev. Lett. 96, 047009 (2006).

[6] M. S. Grønsleth, J. Linder, J.-M. Børven, and A. Sudbø, Phys. Rev. Lett. 97, 147002 (2006).

[7] P. M. R. Brydon, B. Kastening, D. K. Morr, and D. Manske, Phys. Rev. B 77, 104504 (2008); P. M. R. Brydon, ibid. 80, 224520 (2009); P. M. R. Brydon, Y. Asano, and C. Timm, ibid. 83, 180504(R) (2011); 85, 139901 (2012).

[8] A. I. Buzdin, Rev. Mod. Phys. 77, 935 (2005).

[9] F. S. Bergeret, A. F. Volkov, and K. B. Efetov, Rev. Mod. Phys. 77, 1321 (2005).

[10] R. S. Keizer, S. T. B. Goennenwein, T. M. Klapwijk, G. Miao, G. Xiao, and A. Gupta, Nature (London) 439, 825 (2006).

[11] T. S. Khaire, M. A. Khasawneh, W. P. Pratt, and N. O. Birge, Phys. Rev. Lett. 104, 137002 (2010).

[12] M. Fogelström, Phys. Rev. B 62, 11812 (2000).

[13] J. C. Cuevas and M. Fogelström, Phys. Rev. B 64, 104502 (2001).

[14] M. Andersson, J. C. Cuevas, and M. Fogelström, Physica C 367, 117 (2002).

[15] D. Huertas-Hernando, Y. V. Nazarov, and W. Belzig, Phys. Rev. Lett. 88, 047003 (2002).

[16] I. V. Bobkova, Phys. Rev. B 73, 012506 (2006); I. V. Bobkova and A. M. Bobkov, ibid. 76, 094517 (2007).

[17] E. Zhao and J. A. Sauls, Phys. Rev. Lett. 98, 206601 (2007).

[18] Z. Shomali, M. Zareyan, and W. Belzig, New J. Phys. 13, 083033 (2011).

[19] J. X. Zhu and A. V. Balatsky, Phys. Rev. B 67, 174505 (2003); J. X. Zhu, Z. Nussinov, A. Shnirman, and A. V. Balatsky, Phys. Rev. Lett. 92, 107001 (2004).

[20] J. Michelsen, V. S. Shumeiko, and G. Wendin, Phys. Rev. B 77, 184506 (2008).

[21] A. F. Volkov and K. B. Efetov, Phys. Rev. Lett. 103, 037003 (2009).

[22] C. Bell, S. Milikisyants, M. Huber, and J. Aarts, Phys. Rev. Lett. 100, 047002 (2008)
[23] J. P. Morten, A. Brataas, G. E. W. Bauer, W. Belzig, and Y. Tserkovnyak, Europhys. Lett. 84, 57008 (2008).

[24] H. J. Skadsem, A. Brataas, J. Martinek, and Y. Tserkovnyak, Phys. Rev. B 84, 104420 (2011).

[25] C. Richard, M. Houzet, and J. S. Meyer, Phys. Rev. Lett. 109, 057002 (2012).

[26] C. Thirion, W. Wernsdorfer, and D. Mailly, Nature Mater. 2, 524 (2003).

[27] I. Petković, M. Aprili, S. E. Barnes, F. Beuneu, and S. Maekawa, Phys. Rev. B 80, 220502 (2009).

[28] S. E. Barnes, M. Aprili, I. Petković, and S. Maekawa, Supercond. Sci. Technol. 24, 024020 (2011).

[29] C. J. Muller, J. M. van Ruitenbeek, and L. J. de Jongh, Phys. Rev. Lett. 69, 140 (1992).

[30] E. Scheer, N. Agrait, J. C. Cuevas, A. Levy-Yeyati, B. Ludolph, A. Martin-Rodero, G. R. Bollinger, J. M. van Ruitenbeek, and C. Urbina, Nature (London) 394, 154 (1998); E. Scheer, W. Belzig, Y. Naveh, M. H. Devoret, D. Esteve, and C. Urbina, Phys. Rev. Lett. 86, 284 (2001).

[31] S. Teber, C. Holmqvist, and M. Fogelström, Phys. Rev. B 81, 174503 (2010).

[32] C. Holmqvist, S. Teber, and M. Fogelström, Phys. Rev. B 83, 104521 (2011); C. Holmqvist, W. Belzig, and M. Fogelström, ibid. 86, 054519 (2012).

[33] H. B. Heersche, Z. de Groot, J. A. Folk, H. S. J. van der Zant, C. Romeike, M. R. Wegewijs, L. Zobbi, D. Barreca, E. Tondello, and A. Cornia, Phys. Rev. Lett. 96, 206801 (2006).

[34] M.-H. Jo, J. E. Grose, K. Baheti, M. M. Deshmukh, J. J. Sokol, E. M. Rumberger, D. N. Hendrickson, J. R. Long, H. Park, and D. C. Ralph, Nano Lett. 6, 2014 (2006).

[35] L. Bogani and W. Wernsdorfer, Nature Mater. 7, 179 (2008).

[36] S. Kahle, Z. Deng, N. Malinowski, C. Tonnoir, A. FormentAliaga, N. Thontasen, G. Rinke, D. Le, V. Turkowski, T. S. Rahman, S. Rauschenbach, M. Ternes, and K. Kern, Nano Lett. 12, 518 (2012).

[37] K. Franke, G. Schulze, and J. I. Pascual, Science 332, 940 (2011).

[38] Z. Nussinov, A. Shnirman, D. P. Arovas, A. V. Balatsky, and J. X. Zhu, Phys. Rev. B 71, 214520 (2005).

[39] A. V. Balatsky, Y. Manassen, and R. Salem, Phys. Rev. B 66, 195416 (2002). 
[40] L. N. Bulaevskii, V. V. Kuzii, and A. A. Sobyanin, JETP Lett. 25, 290 (1977).

[41] J. C. Cuevas, J. Heurich, A. Martín-Rodero, A. Levy Yeyati, and G. Schön, Phys. Rev. Lett. 88, 157001 (2002).

[42] M. Octavio, M. Tinkham, G. E. Blonder, and T. M. Klapwijk, Phys. Rev. B 27, 6739 (1983).

[43] E. N. Bratus, V. S. Shumeiko, and G. Wendin, Phys. Rev. Lett. 74, 2110 (1995).

[44] D. Averin and A. Bardas, Phys. Rev. Lett. 75, 1831 (1995).

[45] J. C. Cuevas, A. Martín-Rodero, and A. Levy Yeyati, Phys. Rev. B 54, 7366 (1996).

[46] L. Cai and E. M. Chudnovsky, Phys. Rev. B 82, 104429 (2010).
[47] J. Kopu, M. Eschrig, J. C. Cuevas, and M. Fogelström, Phys. Rev. B 69, 094501 (2004)

[48] G. Eilenberger, Z. Phys. 214, 195 (1968).

[49] A. I. Larkin and Y. N. Ovchinnikov, Zh. Eksp. Teor. Fiz. 55, 2262 (1968) [Sov. Phys. JETP 28, 1200 (1969)].

[50] G. M. Eliashberg, Zh. Eksp. Teor. Fiz. 61, 1254 (1971) [Sov. Phys. JETP 34, 668 (1972)].

[51] J. W. Serene and D. Rainer, Phys. Rep. 101, 221 (1983).

[52] K. Nagai and J. Hara, J. Low. Temp. Phys. 71, 351 (1988).

[53] Y. Uzawa and Z. Wang, Phys. Rev. Lett. 95, 017002 (2005).

[54] M. Chauvin, P. vom Stein, H. Pothier, P. Joyez, M. E. Huber, D. Esteve, and C. Urbina, Phys. Rev. Lett. 97, 067006 (2006). 